\title{
Kinematics of extreme waves in deep water
}

\author{
John Grue*, Didier Clamond, Morten Huseby, Atle Jensen \\ Mechanics Division, Department of Mathematics, Univeristy of Oslo, P.O. Box 1053, Blindern, Oslo 0316, Norway
}

Received 1 January 2003; revised 2 February 2004; accepted 1 March 2004

Available online 20 June 2004

\begin{abstract}
The velocity profiles under crest of a total of 62 different steep wave events in deep water are measured in laboratory using particle image velocimetry. The waves take place in the leading unsteady part of a wave train, focusing wave fields and random wave series. Complementary fully nonlinear theoretical/numerical wave computations are performed. The experimental velocities have been put on a nondimensional form in the following way: from the wave record (at a fixed point) the (local) trough-to-trough period, $T_{\mathrm{TT}}$ and the maximal elevation above mean water level, $\eta_{\mathrm{m}}$ of an individual steep wave event are identified. The local wavenumber, $k$ and an estimate of the wave slope, $\epsilon$ are evaluated from $\omega^{2} /(g k)=1+\epsilon^{2}, k \eta_{\mathrm{m}}=\epsilon+\frac{1}{2} \epsilon^{2}+\frac{1}{2} \epsilon^{3}$, where $\omega=2 \pi / T_{\mathrm{TT}}$ and $g$ denotes the acceleration of gravity. A reference fluid velocity, $\epsilon \sqrt{g / k}$ is then defined. Deep water waves with a fluid velocity up to $75 \%$ of the estimated wave speed are measured. The corresponding $k \eta_{\mathrm{m}}$ is 0.62 . A strong collapse of the nondimensional experimental velocity profiles is found. This is also true with the fully nonlinear computations of transient waves. There is excellent agreement between the present measurements and previously published Laser Doppler Anemometry data. A surprising result, obtained by comparison, is that the nondimensional experimental velocities fit with the exponential profile, i.e. $\mathrm{e}^{k y}, y$ the vertical coordinate, with $y=0$ in the mean water level.

(C) 2004 Elsevier Ltd. All rights reserved.
\end{abstract}

Keywords: Wave kinematics; Extreme waves; PIV

\section{Introduction}

Enhanced evidence and description of the kinematics during steep wave events at sea are requested by the offshore and ocean engineering industry. The velocities in steep waves are required for subsequent analysis of loads on, e.g. ships, offshore platforms, tension legs and risers. Despite the numerous studies on the subject, proper knowledge of kinematics of steep irregular ocean waves is still lacking. This provides the motivation of the present investigation. We compare experimental velocity fields due to sets of random wave trains, steep wave events due to focusing waves, steep wave events due to the unsteady leading part of a periodic wave train, and the velocities in computations of steep transient waves. We compare precise Particle Image Velocimetry (PIV) measurements in laboratory and a fully nonlinear modeling. The velocity immediately below the wave crest is focused.

While irrotational flow theory may predict the wave kinematics up to breaking, the theory has shortcomings

\footnotetext{
* Corresponding author. Tel.: +47-2285-5839; fax: +47-2285-4349.

E-mail address: johng@math.uio.no (J. Grue).
}

beyond this limit. This is where experiments become particularly valuable since they are not limited by wave breaking. For example, a series of breaking wave events may take place during the long irregular wave tests undertaken here, recording several strong wave events.

\subsection{Previous experimental works}

We begin with a short summary of previous experimental work. Large scale observations of the kinematics of storm waves are given by, e.g. Buckley and Stavovy [1] and Forristall [2]. LDV (Laser Doppler Velocimetry) laboratory experiments are carried out, most notably by Skjelbreia et al. [3,4], Kim et al. [5], Longridge et al. [6] and Baldock et al. [7]. These works include descriptions of theoretical models.

In the large scale FULWACK experiment [2], the main purpose was to obtain velocity measurements at locations relatively high-up in the waves. They used current meters located at 26, 16, $6 \mathrm{ft}(8.2,5,1.9 \mathrm{~m})$ above mean sea level. The largest observed speed at the top current meter was $20.62 \mathrm{ft} / \mathrm{s}(6.5 \mathrm{~m} / \mathrm{s})$.

Kim et al. [5] measured the kinematics due to focusing waves in a laboratory wave tank with moderately deep water 
using LDA (Laser Doppler Anemometry). Recordings were made at three levels between the mean water line and the crest. Their most extreme wave had an estimated wave slope of 0.28 . Very large horizontal velocity was documented at the crest of the wave, up to $64 \%$ of the measured phase velocity. (The effect of a finite water depth is rather pronounced in the experiments.) They also estimated the acceleration field including the convective acceleration using finite difference technique. Comparisons were made with stretching methods, which they found to greatly underestimate the fluid velocity at the crest of the transient wave.

LDV measurements of steep wave events of an irregular sea, at several vertical positions of the wave field, were performed by Skjelbreia et al. [3,4]. The local wave slope was up to about 0.25 . Major emphasis was given to the surface zone. They measured the fluid velocity at levels close to the crest, half way and one-quarter way between the mean water level and crest, concluding that the stretching method of Wheeler [8] compared better with the measurements of the horizontal velocity below wave crests than Stokes' fifth-order model. No accelerations were obtained. Their experiments were related to small and moderate waves-with relatively few measurements above mean water level. (Our results do not support the Wheeler stretching method.)

Laboratory measurements of wave kinematics of four irregular wave trains using LDA were described by Longridge et al. [6]. They compared the results with a hybrid wave model. The irregular wave fields were produced using the Pierson-Moskowitz (PM) and the JONSWAP spectra. The local wave slope in the wave events they considered was in the range $0.11-0.19$. The paper focused on the velocity and acceleration fields induced by relatively steep wave events. The fluid velocity was measured up to about half way between the mean water level and the crest. The measurements were compared with linear extrapolation, Wheeler stretching and a hybrid wave model, finding that the linear extrapolation was the superior one for the waves with strongest nonlinearity. Accelerations were obtained below the mean water line.

Baldock et al. [7] performed rather detailed and precise sets of wave tank measurements of focusing waves. Both the surface elevation and the induced fluid velocities were presented. The fluid velocities were obtained using LDA. We estimate the wave slope of their largest wave to be about 0.29 (using the procedure given in Section 2.4). Baldock et al. noted that their measured velocities close to the wave crest significantly exceeded the previous velocity recordings by, e.g. Skjelbreia et al. [3,4]. They further noted that their measurements could not be explained by stretching models, for example. (We find here an excellent agreement between the present PIV measurements of several sets of steep wave events and the LDA measurements documented by Baldock et al.)

\subsection{Plunging breakers}

Strong plungers were observed in the experimental works by $\mathrm{Kim}$ et al. [5] $\left(k h=2.0, k \eta_{\mathrm{m}}=0.33\right.$, where $k$ denotes wavenumber, $h$ the water depth and $\eta_{\mathrm{m}}$ the maximal elevation of the event) and Skyner [9] $(k h=2.6$, $\left.k \eta_{\mathrm{m}}=0.36\right)$. In the experiments by Skyner the recorded fluid velocity was found to exceed the (nonlinear) wave speed, although the latter is not well defined for strongly nonlinear waves. We note that the velocity field beneath unsteady two-dimensional water waves have been accurately computed up to the commencement of overturning by Dold and Peregrine [10] and Peregrine [11]. Recent experiments on a spilling breaker show that some water exceeds the wave speed, but that the mean velocity may well be smaller [12].

Although plunging breakers are not so common in deep water, these may occur. In the steepest wave event we have observed in the present experiments, the value of $k h$ is 5 . This wave is developing into a weak breaker. The estimated local wavenumber times maximal elevation of the event is $k \eta_{\mathrm{m}}=0.62$ and the largest measured fluid velocity under crest is $75 \%$ of the phase velocity. Data for steep wave events are compared in Section 5.2.

\subsection{Shortcomings of previous work and points requested}

Theories of steady and random wave fields were reviewed by Gudmestad [13] concluding that regular waves are very accurately predicted by nonlinear theory. He noted that the kinematics in random seas, or in sea states where unsteady very steep waves occur, is less well predicted, however. Shortcomings of previous work and main points requested include: there is lack of resolution in velocity field measurements above the mean water level, particularly in the top and near the tip of the wave. Denser velocity measurements are required to reduce the uncertainty in the acceleration estimates. More laboratory (and full scale) measurements are requested to complement the relatively scarce selection of waves that are studied in previous works.

An important point is that most of the experiments so far have been carried out for relatively small or moderately steep waves. Several of these measurements are found to compare well with predictions using the second-order model for irregular seas [14] or extensions of this model [15-17]. A good agreement between model and experiment is not seen for very steep waves, however.

The Wheeler stretching method, used e.g. in engineering practice in the Gulf of Mexico, is concluded to give relevant predictions of the horizontal velocity below a wave crest. The practice is based on the experimental results by Skjelbreia et al. [3,4]. In contrast to this, Gudmestad [13] noted that velocities under steep random waves compare less well with the stretching models. Measurements then show larger velocities under the crest than predicted by 
models. According to Gudmestad, the kinematics of freak waves and waves which are near breaking, is not well predicted by any existing theory, and that further research is needed with respect to the kinematics of these waves. A model that takes into account the fully nonlinear wave-wave interaction is requested.

\subsection{Focus of the present work}

Our focus is the kinematics of steep wave events which may occur on the surface of the ocean. We compare theoretical and numerical predictions using a recent fully nonlinear wave model (which has no limitations with regard to nonlinearity, dispersion or resolution) with high resolution PIV experiments in a laboratory wave tank. We consider several different wave scenarios in a total of 62 different steep wave events in the wave tank. The total number of PIV/wave experiments reported here is 122 , counting all repetitions which are carried out. Our main objective is to identify the velocity profile under crest of a steep wave event. The wave event is a result of a process on the surface of the fluid where the wave field, from an initial state, has developed due to nonlinearity and dispersion. We assume that this process has gone on during sufficiently long time such that the initial conditions are 'forgotten'. We here focus on the kinematics of several steep wave events resulting from four different generation procedures. Three of the procedures are pursued experimentally in the laboratory. A fourth one results from numerical simulations.

Most of the experimental waves can be considered to be deep water waves ( $k h$ larger than about 3 ). In a few of the experiments the finite depth of the water introduces a small, systematic change in the wave profiles compared to the deep water results, however. This typically occurs for $k h \sim 2$ and is discussed in Section 4.3.3.

We compare our measurements with those in Refs. [5,7, 9]. We further discuss the large-scale observations of the FULWACK experiment in view of the present results, see Section 5. The waves studied here are significantly larger than the waves documented in Refs. $[3,4,6]$, and comparisons with these works are not performed.

We aim at a general way to characterize the steep wave events in the experiments. The purpose is to interpret and communicate the results. More precisely, from the wave record we identify a local wave period and a maximal excursion of an individual steep wave event. From these two parameters we provide an estimate of the local wave slope, the wavenumber and a characteristic fluid velocity of the event. The procedure is documented in full in Section 2.4.

\section{Theoretical models}

Third-order Stokes waves, fully nonlinear simulations of Stokes waves and a general fully nonlinear, unsteady wave model provide references for the experiments. The models are first discussed.

\subsection{Third-order Stokes waves}

The theory of third-order Stokes waves is valid for periodic progressive irrotational wave motion with a moderate wave slope in deep water. Let $\{x, y, t\}$ be the horizontal, upward vertical and time variables, respectively, and let the mean water level be at $y=0$ and the free surface at $y=\eta$. Let $\phi$ denote the velocity potential such that $\nabla \phi \rightarrow 0$ as $y \rightarrow-\infty$. Denoting $\{g, k, \omega\}$ the acceleration due to gravity, the wavenumber and the angular frequency, a variant of Stokes' third-order approximation reads

$$
\begin{aligned}
\frac{k \phi}{\sqrt{g / k}}=\epsilon \mathrm{e}^{k y} \sin \theta+\mathrm{O}\left(\epsilon^{4}\right), & \\
k \eta= & \left(1+\frac{1}{8} \epsilon^{2}\right) \epsilon \cos \theta+\frac{1}{2} \epsilon^{2} \cos 2 \theta \\
& +\frac{3}{8} \epsilon^{3} \cos 3 \theta+\mathrm{O}\left(\epsilon^{4}\right), \\
\frac{\omega^{2}}{g k}= & 1+\epsilon^{2}+\mathrm{O}\left(\epsilon^{3}\right), \\
k \eta_{\mathrm{m}}= & \epsilon+\frac{1}{2} \epsilon^{2}+\frac{1}{2} \epsilon^{2}+\mathrm{O}\left(\epsilon^{4}\right),
\end{aligned}
$$

where $\theta=k x-\omega t$ denotes the phase function and the latter equation relates $\epsilon$ to the maximum surface elevation times the wavenumber. (In the expansion presented here the amplitude of the fundamental frequency of the fluid velocity is chosen as small parameter. The half of the wave height is determined by $\left[\epsilon+\frac{1}{2} \epsilon^{3}+\mathrm{O}\left(\epsilon^{4}\right)\right] / k$.)

Eq. (1) shows that an exact Stokes wave velocity field is well approximated by linear theory, even for relatively steep waves. For this reason, linear wave theory is widely used to estimate the wave induced velocity field above and below the mean water level. Indeed, the approximated velocity potential (1) suggests that the velocity field below the crest is almost self-similar (within a reasonable accuracy). It is tempting to evaluate a dimensionless horizontal velocity below the wave crest of the form $u /(\epsilon \sqrt{g / k})$, where $u$ denotes the horizontal velocity.

\subsection{Fully nonlinear Stokes waves}

Exact (fully nonlinear) computations of (steady) Stokes waves are included for illustrative purposes. The computations are facilitated by Fenton's program [18] (providing an accuracy of at least five digits for the results shown here). The computations in Fig. 1a of the velocity profile below crest are very close to the exponential profile when the wave is moderately steep ( $\epsilon$ up to about 0.2 ). Such profiles have also been experimentally obtained in PIV experiments for wave slope up to 0.16 [19]. The theoretical results are far from uniform when $\epsilon$ is in the range $0.27-0.36$ (Fig. 1a). 

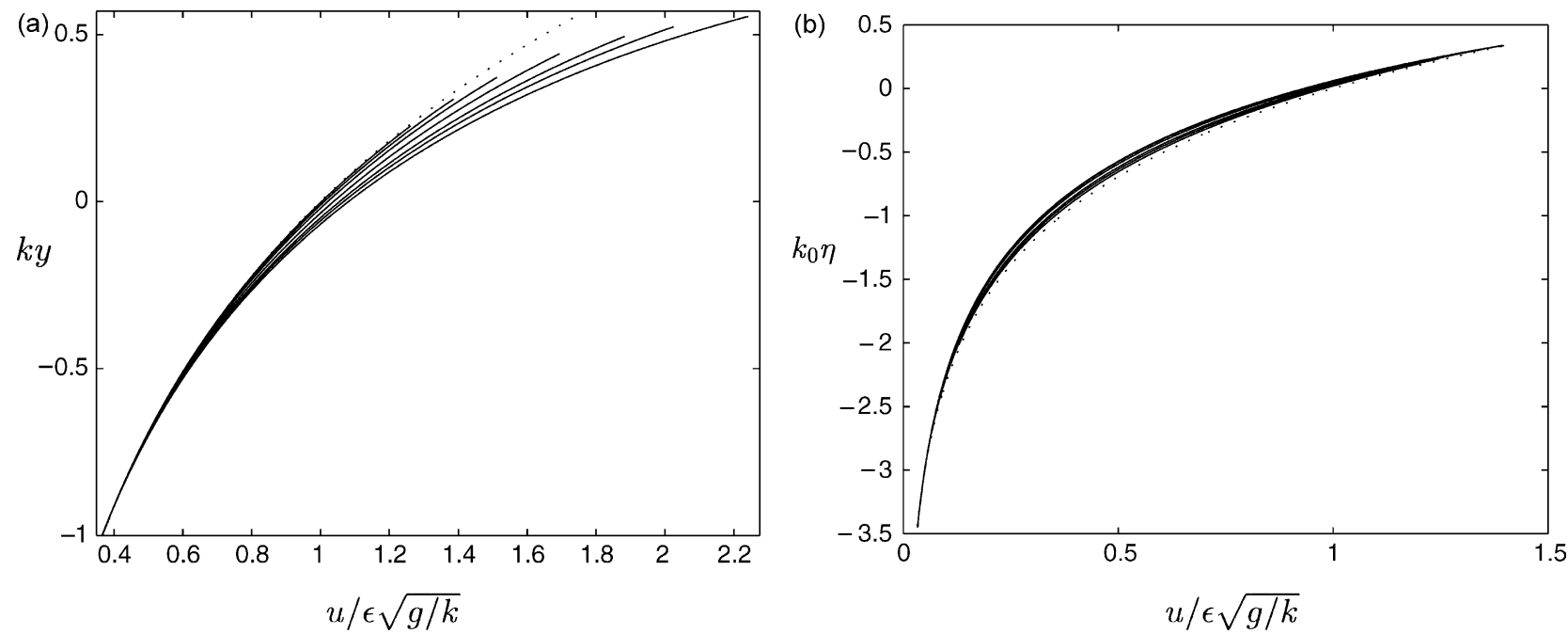

Fig. 1. Normalized velocity profiles in fully nonlinear Stokes waves (computed as described in Section 2.2) (a) and freak waves obtained (computed as described in Sections 2.3) (b). Values of $\epsilon$ in plot (a) (from largest to smallest): 0.360, 0.357, 0.351, 0.333, 0.298, 0.258, 0.201. Values of $\epsilon$ in plot (b): 0.290, $0.290,0.285,0.267,0.236,0.212,0.182$. Dotted line: $\mathrm{e}^{k y}$.

We shall see below that the nondimensional experimental velocities, with $k \eta_{\mathrm{m}}$ up to 0.62 (and $\epsilon$ up to 0.46 ), agree better with estimates from Stokes third-order solution than with the fully nonlinear Stokes solution, for the steep waves. The trick is to identify a local $k$ and $\epsilon$ of the unsteady wave using the procedure of Section 2.4.

\subsection{Fully nonlinear simulations of unsteady waves}

In recent computations, employing a rapid fully nonlinear transient method, very steep wave events have been obtained [20-22]. In brief, the theoretical/numerical scheme integrates the prognostic equations. These result from the kinematic and dynamic boundary conditions at the free surface, i.e.

$\eta_{\mathrm{t}}+\tilde{\psi}_{x}=0, \quad \tilde{\phi}_{\mathrm{t}}+g \eta+\frac{1}{2} \frac{\tilde{\phi}_{x}^{2}-\tilde{\psi}_{x}^{2}+2 \eta_{x} \tilde{\phi}_{x} \tilde{\psi}_{x}}{1+\eta_{x}^{2}}=0$,

where the 'tildes' denote the quantities evaluated at the free surface $y=\eta$, and $\psi$ denotes the stream function. The equation connecting the (scaled) normal velocity at the free surface, $\tilde{\psi}_{x}, \eta$ and $\tilde{\phi}_{x}$, is obtained by solving the Laplace equation in the fluid domain. The resulting equation is on the form

$$
\begin{aligned}
\tilde{\psi}_{x}= & \mathscr{H}\left\{\tilde{\phi}_{x}\right\}+\partial_{x}\left\{\eta \tilde{\phi}_{x}\right\}+\partial_{x}\left\{\mathscr{H}\left\{\eta \mathscr{H}\left\{\tilde{\phi}_{x}\right\}\right\}\right\} \\
& +\mathscr{H}\left\{\frac{1}{\pi} \int_{-\infty}^{\infty} \frac{D^{2}\left(D-\eta_{x}\right) \tilde{\phi}_{x}^{\prime}}{1+D^{2}} \frac{\mathrm{d} x^{\prime}}{x^{\prime}-x}\right. \\
& \left.-\frac{1}{\pi} \int_{-\infty}^{\infty} \frac{D\left(D-\eta_{x}\right) \tilde{\psi}_{x}^{\prime}}{1+D^{2}} \frac{\mathrm{d} x^{\prime}}{x^{\prime}-x}\right\}
\end{aligned}
$$

where $\mathscr{H}$ denotes Hilbert transform, $D=\left(\eta^{\prime}-\eta\right) /\left(x^{\prime}-x\right)$, $\tilde{\phi}=\tilde{\phi}(x, t), \tilde{\phi}^{\prime}=\tilde{\phi}\left(x^{\prime}, t\right)$, etc. The latter implicit equation can be solved numerically very quickly via Fast Fourier Transform and truncated integrations.
Numerical simulations of very steep (freak) waves are obtained as in Refs. [21,22]: the initial state of the wave field is specified into two steps. First an exact steady Stokes wave, with wavenumber $k_{0}$ and amplitude $a_{0}$ ( $a_{0}$ half the total wave height) was computed. Secondly, the surface elevation and the tangential velocity at the surface were multiplied by the 'bell' function such $\left[\epsilon_{0} \sqrt{2} a_{0} k_{0}^{2}\left(x-x_{0}\right)\right]$, where the parameter $\epsilon_{0}$ determines the length of the packet. The case $\epsilon_{0}=1$ corresponds to an exact soliton solution of the nonlinear Schrödinger equation.

This initial condition, in the form of a localized wave packet, is input to the fully nonlinear numerical scheme. With $a_{0} k_{0}=0.091$ and $\epsilon_{0}=0.263$ a very steep wave event is produced after 155 wave periods. The maximal wave elevation $\eta_{\mathrm{m}}$ times $k_{0}$ is then $k_{0} \eta_{\mathrm{m}}=0.29$. Other relatively steep wave events (with smaller $k_{0} \eta_{\mathrm{m}}$ ) are produced using the same procedure, with less strong initial conditions. The computational domain involves 128 wavelengths and the carrier wave is discretized over 32 nodes per wavelength. This means that all harmonics up to the 15th are resolved, and that 128 Fourier modes are included in the spectral band $\left[k_{0}-\frac{1}{2} k_{0} ; k_{0}+\frac{1}{2} k_{0}\right]$.

The wave induced Stokes drift close to the free surface is inherent in the fully nonlinear formulation. This is also true with regard to a return flow beneath the wave group. The latter contributes to an almost vanishing time-averaged horizontal mass flux during the wave motion. This is indicated in the fully nonlinear simulations by the integral of the stream function along the free surface, i.e. $\int_{-\infty}^{\infty} \tilde{\psi} \times$ $\mathrm{d} x=\int_{-\infty}^{\infty} \int_{-\infty}^{\eta} u \mathrm{~d} y \mathrm{~d} x$. This integral is an order of magnitude smaller than the estimated Stokes drift indicating that a return flow beneath the wave group balances the Stokes drift, approximately. The return flow is caused by the radiation stress [14] and is reproduced in second-order theories [23-25]. 


\subsection{Velocity profile on nondimensional form}

We are seeking a nondimensional representation of the velocity profile under crest of a steep unsteady wave event. It is tempting to test out the following procedure:

1. In a steep wave event we identify, from the time history of the surface elevation at a fixed geometrical location, the trough-to-trough period, $T_{\mathrm{TT}}$ and the maximal elevation, $\eta_{\mathrm{m}}$ of the event. We then define the local angular frequency by $\omega=2 \pi / T_{\mathrm{TT}}$.

2. We next compute the wavenumber $k$ and a measure of the wave slope $\epsilon$ solving numerically the system of equations

$$
\omega^{2} /(g k)=1+\epsilon^{2}, \quad k \eta_{\mathrm{m}}=\epsilon+\frac{1}{2} \epsilon^{2}+\frac{1}{2} \epsilon^{3},
$$

which result from a truncation of Eqs. (3) and (4). The half of the wave height is estimated by $\left[\epsilon+\frac{1}{2} \epsilon^{3}\right] / k$. Such a procedure makes it possible to extract the local wave length from the time record of the wave elevation in one point. Using the fully numerical simulations we have compared $2 \pi / k$, obtained by this method, with the trough-to-trough wavelength, $L_{\mathrm{TT}}$ obtained directly from the nonlinear wave computations. Several steep (freak) wave events have been tested. We found a relative difference of about $3.5 \%$ for the larger waves, with estimated $k \eta_{\mathrm{m}}=0.34$. The agreement was better for the smaller waves.

We then consider the horizontal velocity profile below the simulated steep waves. Nondimensional velocity profiles are obtained dividing the fluid velocity by $u_{\text {ref }}=$ $\epsilon \sqrt{g / k}$ using the procedure outlined above. Results from the computations with $\epsilon$ in the range $0.18-0.29$ are presented in Fig. 1b. A collapse of the velocity profiles is observed. Comparison is also made with the exponential profile. The nondimensional velocities resulting from the transient wave computations, in the region above mean water line, are closer to the exponential profile than observed in fully nonlinear computations of Stokes waves, when $\epsilon$ is large.

This procedure to obtain nondimensional velocity fields appears relevant to steep, unsteady wave events. It is simple and robust in use. The resulting nondimensional experimental velocities are expected to be relatively close. We have also tested out corresponding equations including terms up to fifth order, finding only very minor corrections.

The main focus here is the velocities above the mean water line, with main emphasis on the flow close to the wave crest. In what follows we use as theoretical reference the largest 'freak' wave computation with $\epsilon=0.29$ and $k \eta_{\mathrm{m}}=$ 0.34 . In addition we compare with the exponential profile. Different procedures to obtain a local wave period have been tested: zero upcrossing, zero downcrossing, etc. It appears, after some tests, that the trough-to-trough period gives the more consistent results.

The characterization of individual waves of the wave field by the local wavenumber and wave period can mathematically be justified when the wave spectrum is sufficiently narrowbanded. Such a procedure represents a first step also in the more general (and broadbanded) case. We have in our laboratory measured the nonlinear dispersion relation in focusing wave groups finding that the measurements of wave period and wave length fit with nonlinear theory of slowly varying wave trains $[26,27]$.

\section{Experimental environment and procedure}

The experiments were carried out in a wave tank $24.6 \mathrm{~m}$ long and $0.5 \mathrm{~m}$ wide in the Hydrodynamics Laboratory at the University of Oslo. It was filled with water to a depth of $0.6 \mathrm{~m}$ in the experiments with the leading wave of a wave group (Section 4.1), and to a depth of $0.72 \mathrm{~m}$ in the focusing wave and random wave experiments (Sections 4.2 and 4.3). In one end of the tank there is a hydraulic piston wavemaker with movements controlled by a computer. The update rate of the wave maker and the sampling rate of the data acquisition is $1000 \mathrm{~Hz}$. At the opposite end of the wave tank there is an absorbing beach which reflects less than $3 \%$ of the amplitude of the incoming waves.

The kinematics of the waves is obtained by employing an extended PIV system [19]. The system, particularly designed to measure accelerations, consists of two CCD cameras, a scanning laser beam and a synchronizer controlled and monitored by a computer. The laser source is a CW argon ion laser, sufficiently powerful $(10 \mathrm{~W})$ to provide light for sequences of recorded image pairs. The two high-sensitive cooled PCO Sensicam cameras have a resolution of $1280 \times 1024$ pixels with 12-bit digital output. A high-speed acousto-optic modulator is used to shut off the CW laser after two scans of the flow have been captured by the first camera. The modulator switches the beam back on for the second velocity measurement after a programmed delay. Synchronization of the cameras and beam modulator with the scanning beam system is achieved with a purposebuilt multi-channel synchronizer device and operated from an integrated modular tree based acquisition and processing software system. We present here velocity measurements from the first camera.

First, the wave elevation of the actual wave field was recorded at specified locations using wave gauges. Individual steep wave events were then identified from the wave records. Each experiment was repeated measuring the velocity field under the large wave(s) using PIV. The system was triggered at the time instant when the wave crest was in the middle of the interrogation window. Several repetitions with the same wave conditions were performed. The water in the tank was seeded with polyamid particles with diameter approximately $50 \mu \mathrm{m}$. The field of view was 
$25 \mathrm{~cm} \times 20 \mathrm{~cm}$ in all the runs. In most experiments an interrogation window of $64 \times 64$ pixels with an overlap of $50 \%$ was used. In some experiments the interrogation window was $32 \times 32$ pixels. More details of the PIV system and the data processing procedures may be found in Ref. [19].

We obtain from the velocimetry the velocity field of the waves in vicinity of the crest. The wave events are characterized by the local period and the local maximal elevation, both obtained from the wave record as described in Section 2.4. The parameter $\epsilon$, wavenumber and characteristic velocity are obtained from Eq. (5). Nondimensional velocities are then evaluated. Physical velocities are obtained from the presented results multiplying by $\epsilon \sqrt{g / k}$.

\section{Experimental results}

\subsection{Leading wave of a wave train}

A sinusoidal motion of the wave maker with frequency $\omega_{0}$ generates a wave train which has a transient leading part followed by periodic waves. (The motion of the wave maker has in these experiments a constant amplitude, apart from a short initial period of $1 \mathrm{~s}$ when an amplitude function rises from 0 to the value of 0.99 . The amplitude function has the form of a tanh-function.) We pay here attention to the transient leading part of the wave fields. Relatively strong unsteady wave events take place there. An example is visualized in Fig. 2a. We investigate the kinematics of all of the largest wave events that are recorded in these series of

(a)

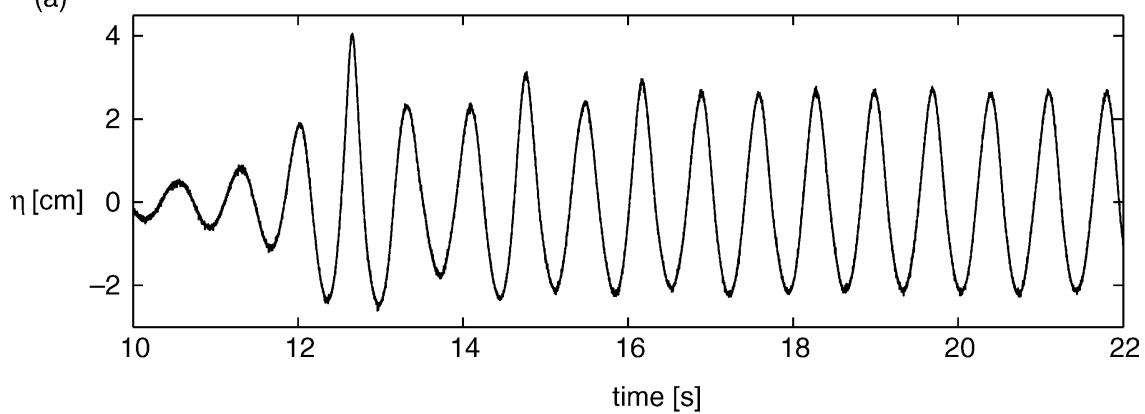

(b)

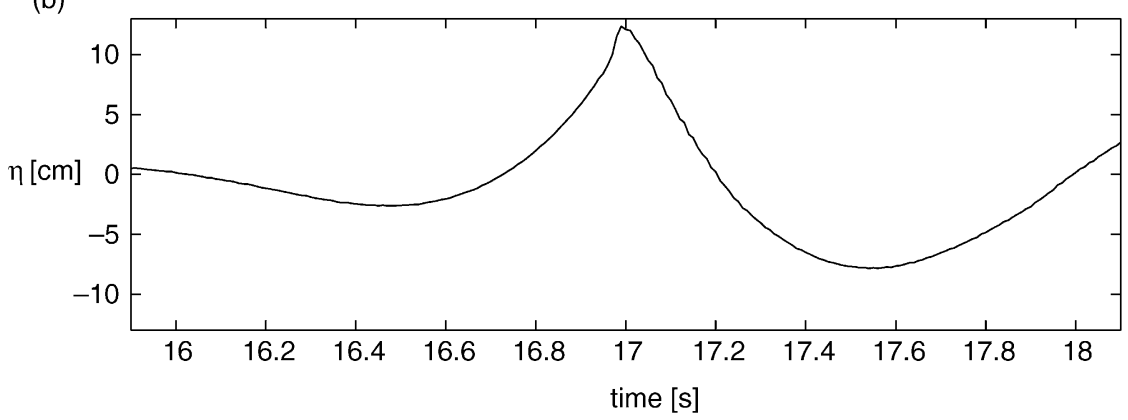

(c)

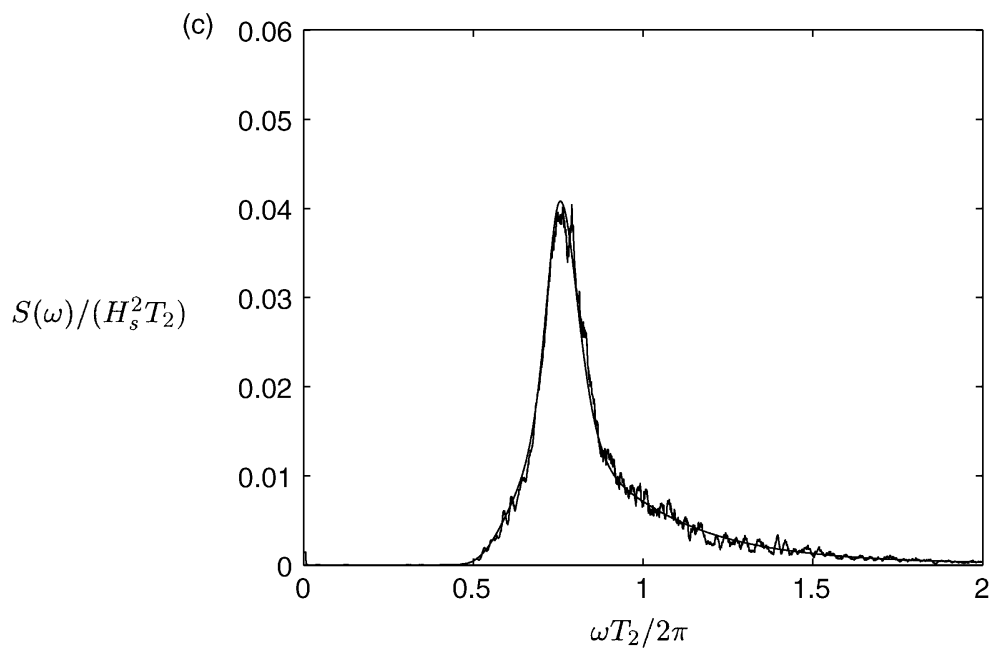

Fig. 2. Wave fields. (a) Leading wave of a wave group. (b) Focusing wave. (c) Spectrum of random waves obtained from wave record vs. input spectrum (smooth line). $\omega_{\mathrm{p}}^{2} H_{\mathrm{s}} / 2 g=0.10, T_{\mathrm{p}}=1.04 \mathrm{~s},\left(T_{2}=0.777 T_{\mathrm{p}}\right)$. 
experiments, counting up to 13 in total. First the wave elevation at a specified location was recorded using a wave gauge. Next the run was repeated measuring the velocity field under the wave using PIV.

In this set of experiments, the parameter $\epsilon$ in the different events is in the range $0.21-0.35$, the inverse of the wave period in the range $0.94-1.49 \mathrm{~s}^{-1}$, and the wavenumber
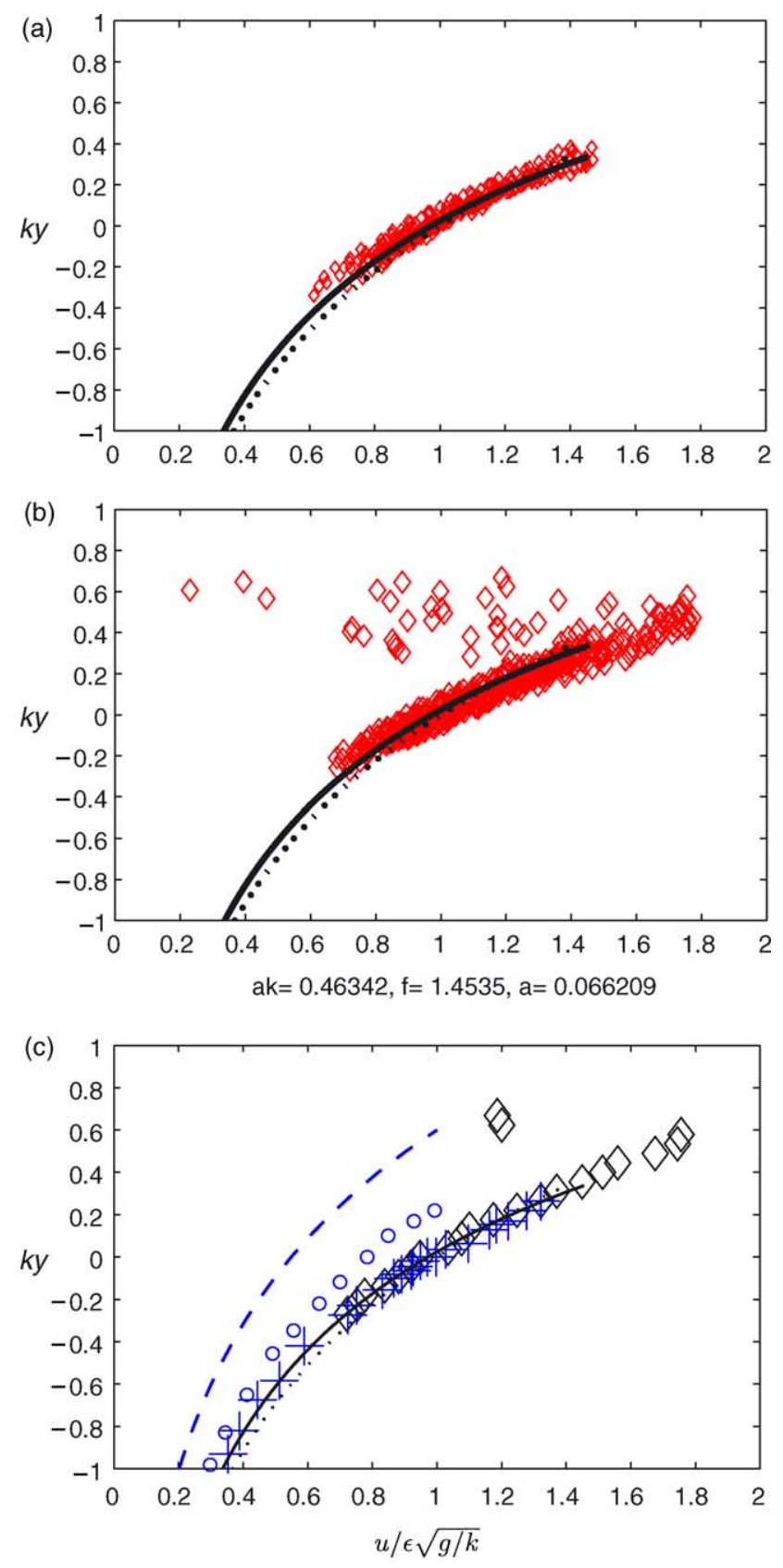

Fig. 3. Horizontal velocity profiles below wave crest. (a) Thirteen large wave events in the leading unsteady part of a wave group, with $0.213<$ $\epsilon<0.348$. (b) Nineteen large wave events of focusing wave groups, with $0.217<\epsilon<0.463$. (c) Largest focusing wave event in: present measurements, $\epsilon=0.46, k \eta_{\mathrm{m}}=0.6(\diamond)$, LDA measurements by Baldock et al. [7] $(+)$, second-order model predictions reproduced from Ref. [7] (O). Solid line: Velocity profile in 'freak' wave computation with $\epsilon=0.29$. Dotted line: $\mathrm{e}^{k y}$. Dashed line: Wheeler method for wave with $\epsilon=0.46, k \eta_{\mathrm{m}}=0.6$. times the water depth, $k h$ in the range $2-5(h=0.6 \mathrm{~m})$. The nondimensional velocity profiles of the 13 different waves are plotted together in Fig. 3a. A surprising collapse of the data is observed. There is only very small scatter in the results. The experimental profiles, put on nondimensional form, are seen to follow the velocity profile of the theoretical freak wave computed in Sections 2.3 and 2.4 (Fig. $1 \mathrm{~b}$ and c). It is also good fit between the experiments and the exponential profile.

\subsection{Focusing waves}

Several sets of focusing waves have been generated in the wave tank. In these runs the water depth was $0.72 \mathrm{~m}$. The motion of the wave maker was given by $\xi(t)=a(t)$ $\sin [\omega(t) t]$, where $\omega(t) / 2 \pi$ was decreased linearly from 1.6 to $0.96 \mathrm{~s}^{-1}$. The amplitude function had the form of $a(t)=$ $\hat{a}_{0} \times\left(2^{8} / 3^{3}\right) \times\left[\left(t / t_{\mathrm{s}}\right)^{3}-\left(t / t_{\mathrm{s}}\right)^{4}\right]$, with $t / t_{\mathrm{s}}<1$, where $t_{\mathrm{s}}$ was in the range 16-26.5 s. $\left(\hat{a}_{0}\right.$ denotes a constant amplitude which could be varied from experiment to experiment.) The large waves were recorded at a time instant the interval $17-21.3 \mathrm{~s}$ after the wave maker motion was started. An example of a steep wave is visualized in Fig. $2 b$.

A total of 19 different focusing wave events were investigated. The parameter $\epsilon$ (see Eq. (5)) in the different events is in the range $0.22-0.46$, the inverse of the wave period in the range $0.80-1.45 \mathrm{~s}^{-1}$, and the wavenumber times the water depth $k h$ in the range $1.8-5(h=0.72 \mathrm{~m})$. (The longest waves in these runs are not deep water waves. The steepest waves, that are the most interesting ones, are deep water waves, however.) The nondimensional velocity profiles of the 19 different wave events are plotted together in Fig. 3b. There is a strong collapse of the data. The experimental profiles follow the velocity profile of the theoretical freak wave computed in Sections 2.3 and 2.4 (Fig. 1b and c). Further, a good comparison between the nondimensional velocities and $\mathrm{e}^{k y}$ is seen. There is only very small scatter in the experimental results. We continue in Section 5 below a discussion of the very large velocities recorded in the focusing wave events. We find that the fluid velocity is up to about $75 \%$ of the estimated phase speed of the wave, with corresponding $k \eta_{\mathrm{m}}=0.62$ (and $k h=5$ ).

Some spurious velocity vectors appear in some of the measurements close to the free surface. These measurements indicate occurrence of breaking in the top of the wave. The breaking processes observed in the experiments will not be discussed further here. We note that the velocity estimates in the thin breaking zones are uncertain.

\subsection{Random waves}

Steep wave events in random seas using the JONSWAP spectrum are then measured. The spectrum is characterized by the significant wave height, $H_{\mathrm{s}}$, the peak period, $T_{\mathrm{p}}$, and the peak enhancement factor, $\gamma=3.3$. A total of six 
different time series were produced, each with a length of $10 \mathrm{~min}$. The input spectrum of the wave field compare excellent with the spectrum produced from the recorded elevation in the tank (Fig. 2c). An effect of a small reflection from the beach is not visible in the figure.

The velocity fields in a total of five steep wave events in each of the six series have been analysed using PIV. The ranges of the parameter, $\boldsymbol{\epsilon}$, inverse period, $f$, and wavenumber of the individual waves times the water depth, $k h$, are indicated in the table below (where $\omega_{\mathrm{p}}=2$ $\left.\pi / T_{\mathrm{p}}, h=0.72 \mathrm{~m}\right)$.

\begin{tabular}{lllllll}
\hline & $\begin{array}{l}H_{\mathrm{s}} \\
(\mathrm{cm})\end{array}$ & $T_{\mathrm{p}}(\mathrm{s})$ & $\begin{array}{l}\omega_{\mathrm{p}}^{2} H_{s} \\
/ 2 g\end{array}$ & & $f\left(\mathrm{~s}^{-1}\right)$ & $k h$ \\
\hline Series 1 & 6.55 & 0.939 & 0.15 & $0.19-0.34$ & $1.21-1.30$ & $3.9-4.6$ \\
Series 2 & 9.60 & 1.150 & 0.15 & $0.21-0.34$ & $0.96-1.04$ & $2.4-2.9$ \\
Series 3 & 8.00 & 1.150 & 0.12 & $0.14-0.34$ & $0.82-1.19$ & $1.6-3.7$ \\
Series 4 & 5.45 & 0.939 & 0.12 & $0.26-0.40$ & $1.14-1.41$ & $3.5-4.9$ \\
Series 5 & 6.55 & 1.040 & 0.12 & $0.18-0.31$ & $1.01-1.29$ & $2.9-4.4$ \\
Series 6 & 6.67 & 1.150 & 0.10 & $0.17-0.22$ & $0.98-1.22$ & $2.7-4.2$ \\
\hline
\end{tabular}

\subsubsection{Velocity profiles}

Each of the series in the random wave experiments had three repetitions, all with the same triggering of the PIV system. The experiments were very repeatable. The velocity data from the repeated runs exhibit a spread of about plus/minus one grid point (there are 40 grid points in each direction), except in some of the breaking wave cases. The results from series 1 show an all over collapse of the velocity profiles when they are put on nondimensional form. Moreover, the measurements are very close to the fully nonlinear computations with $\epsilon=0.29$ and the exponential profile, see Fig. 4. The correspondence is true for wave

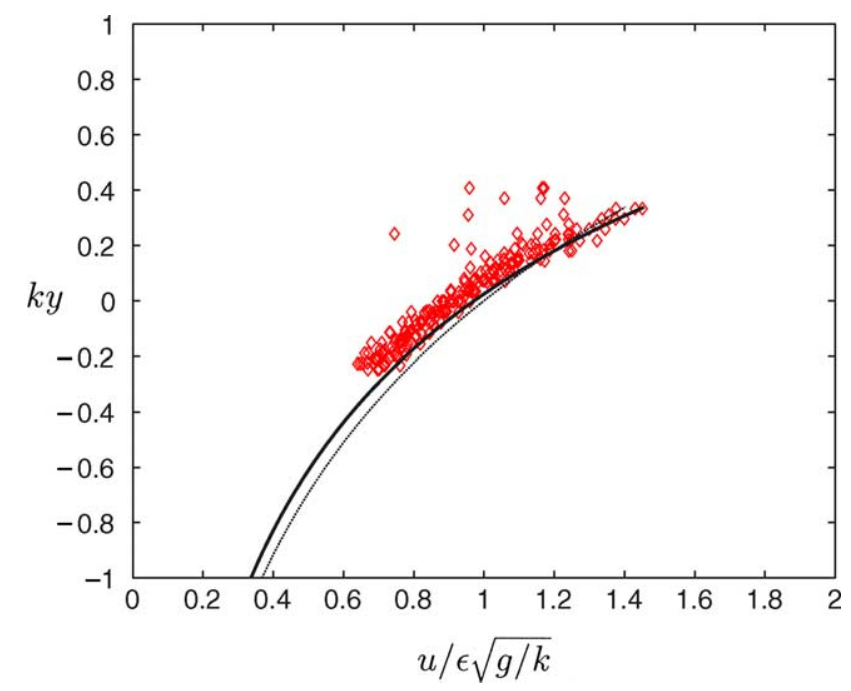

Fig. 4. Horizontal velocity profiles below wave crest. Five wave events of series $1(\diamond)$. Solid line: Velocity profile in 'freak' wave computation with $\epsilon=0.29$. Dotted line: $\mathrm{e}^{k y}$. Recording times: 124.45, 133.24, 165.27, $214.01,283.43 \mathrm{~s}$

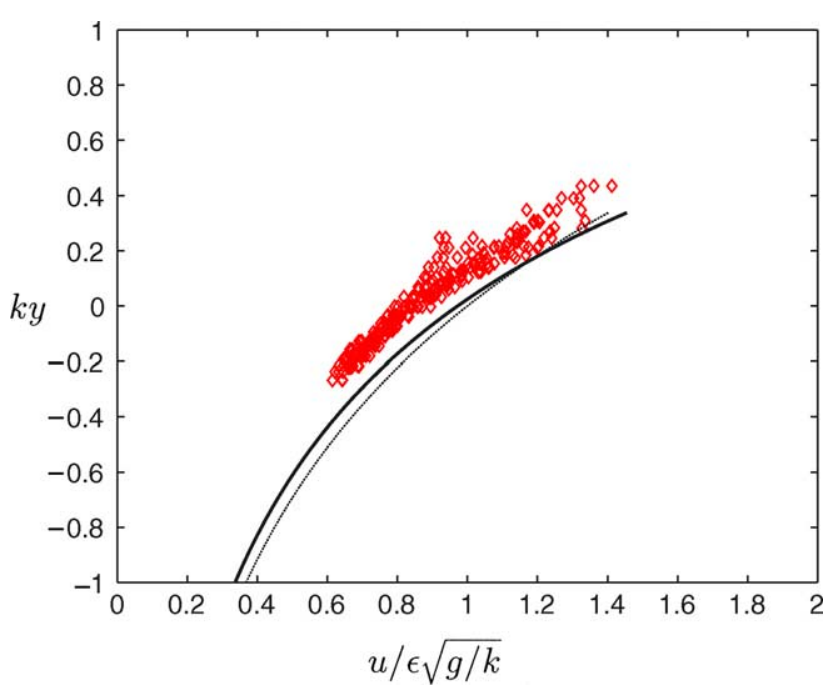

Fig. 5. Same as Fig. 4, but series 4. Recording times: 127.32, 169.76, 256.47, 297.54, $324.72 \mathrm{~s}$.

events that both are early and late in the series and is particularly good in the top of the wave. The events in series 1 are relatively steep, and the water is deep $(0.19<\epsilon<0.34,3.9<k h<4.6)$.

The wave data in series 4 are in many ways comparable to those in series 1 . The nondimensional water depth is in the same range in the two series. The wave slope is somewhat higher in the events in series $4(0.26<\epsilon<0.4)$ than in series 1 . There is again a collapse of the nondimensional velocity data, see Fig. 5. The experimental (nondimensional) velocities are seen to be about $10 \%$ smaller than the fully nonlinear theoretical reference and the exponential profile.

The random wave series number 2 has the same nondimensional value of $\omega_{\mathrm{p}}^{2} H_{\mathrm{s}} / 2 g$ as in series 1 , and the range of the wave slope of the selected wave events is in the same range in the two series. The velocity profiles shown in Fig. 6 are rather close to the observations in series 1 and 4, apart from the velocity profiles in the late part of series 2, i.e. at times $226.9 \mathrm{~s}$ (marked in the figure by $\square$ ) and $304.6 \mathrm{~s}$ (marked by $\diamond$ ). The velocity has then got an additional tilt: the nondimensional velocity near the crest has become larger, and the velocity below the mean water level smaller, than in the previous recordings. The waves are still rather steep waves in deep water. The estimated wave slope in the last recording of series 2 is $\epsilon=0.24$ and the wavenumber times the water depth is $k h=2.9$, for example. The relatively large value of $k h$ indicates that the effect of a finite water depth cannot fully explain the additional tilt.

We note that the waves in series 2 are $40 \%$ longer than those in series 1 and 4 . The wave velocity (group velocity) is correspondingly $20 \%$ higher and the estimated Stokes drift $60 \%$ higher. Correspondingly, an estimated return velocity beneath the wave is $120 \%$ higher in series 2 than in series 1 and 4 . An addition to the return flow due to a finite length of the tank is set up quicker and becomes stronger in series 2 


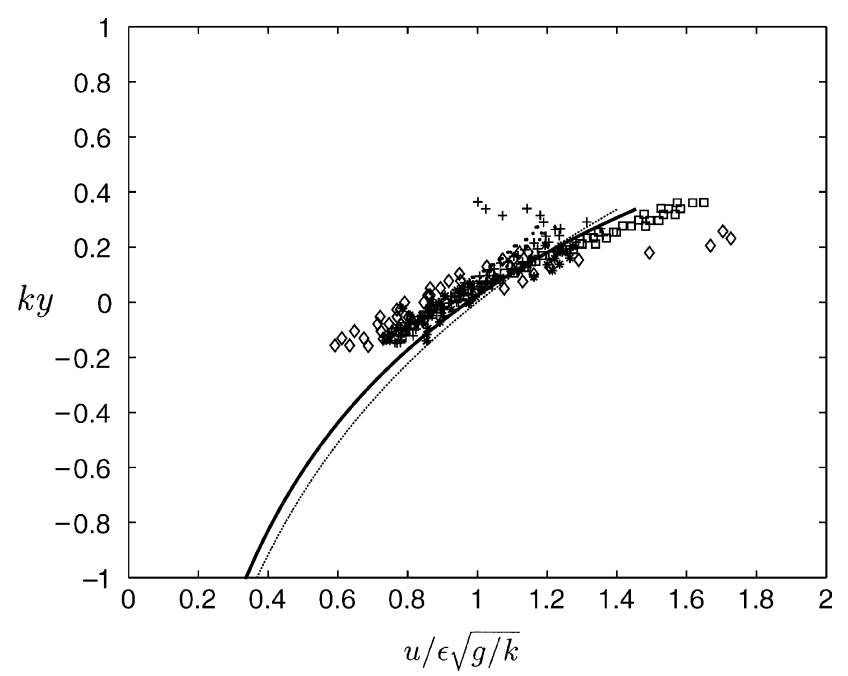

Fig. 6. Horizontal velocity profiles below wave crest. Five wave events of series 2. $\left(t_{1}=124.17 \mathrm{~s}, \epsilon=0.26, k h=2.6, \bullet\right),\left(t_{2}=162.02 \mathrm{~s}, \epsilon=0.21\right.$, $k h=2.6, *), \quad\left(t_{3}=184.45 \mathrm{~s}, \epsilon=0.29, k h=2.7,+\right), \quad\left(t_{4}=226.95 \mathrm{~s}\right.$, $\epsilon=0.34, k h=2.4 \square),\left(t_{5}=304.62 \mathrm{~s}, \epsilon=0.24, k h=2.9, \diamond\right)$. Solid line: Velocity profile in 'freak' wave computation with $\epsilon=0.29$. Dotted line: $\mathrm{e}^{k y}$.

than in 1 and 4 . With the PIV equipment we are able to perform precise measurements of the instantaneous velocity field. Recordings of long time series of the wave field using PIV is prevented by limitations of the apparatus, however, and we are unable to measure the induced drift velocities in the wave tank using the equipment. We are thus left with estimates of a time averaged (or slowly varying) velocity field in the wave tank, as indicated above. We speculate that a relatively pronounced time averaged (slowly varying) return velocity is responsible for the additional tilt of the velocity profiles observed in the late part of the experiments in series 2 .

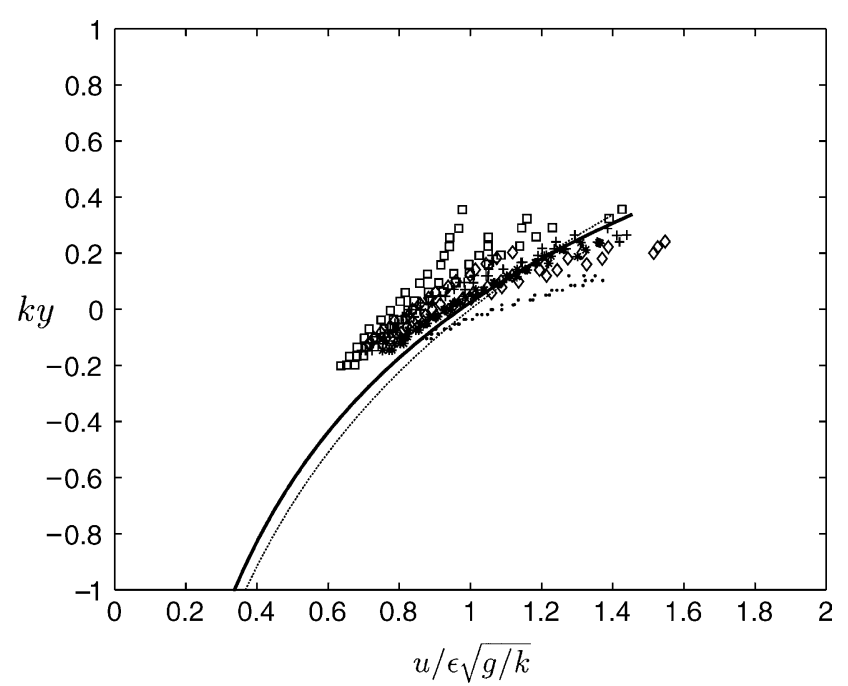

Fig. 7. Same as Fig. 6, but series 3. $\left(t_{1}=117.63 \mathrm{~s}, \epsilon=0.14, k h=1.9\right.$, ○), $\left(t_{2}=166.35 \mathrm{~s}, \epsilon=0.24, k h=2.7, *\right),\left(t_{3}=213.14 \mathrm{~s}, \epsilon=0.26, k h=2.7\right.$, $+),\left(t_{4}=226.90 \mathrm{~s}, \epsilon=0.34, k h=3.7, \square\right),\left(t_{5}=323.57 \mathrm{~s}, \epsilon=0.23, k h=\right.$ $2.3, \diamond)$
The results from series 3 (Fig. 7) show the same tendency as in series 2 apart from the first event in the series (marked by the thin dots) where the local wave has rather small value of $\epsilon=0.14$ and moderate wavenumber $k h=1.9$. The measured nondimensional velocities early in the series are close to the theoretical references (fully nonlinear computation and exponential profile). An additional tilt appears in the velocity profiles late in the runs, however. Some scatter in the data of the fourth wave event (at time $t_{4}=226.9 \mathrm{~s}$ ) is seen.

In series 5 (results not shown) the waves are deep water waves $(k h>2.9)$ and the range of $\epsilon$ is $0.18-0.3$. The data agree with the results from series 1 and 4 , more or less. While some scatter in the data is noted, the spread seems not to be systematic. In series 6 (results not shown), the wave slope is systematically smaller than in the other experimental series $(0.17<\epsilon<0.22)$. The recordings early in the series are close to the theories. Late in the series the velocity profiles become somewhat tilted.

\subsubsection{Very steep wave events}

Results from all steep events in the random wave series, with $\epsilon>0.3$, are plotted together in Fig. 8. The figure shows a relatively systematic appearance of the velocities in steep wave events in random wave fields. The measured profiles above the mean water line, put on nondimensional form, almost solely depend on the local estimated wavenumber and wave slope, when the latter is large. An upper bound of the velocities is given by the fully nonlinear computation and exponential profile, practically speaking. This conclusion is true also for events in late parts of the time series when there is evidence of an enhanced return flow due to the finite length of the wave tank. This conclusion also holds even if there is some (small) effect of a finite water depth $(k h=2.4$ in one of the runs).

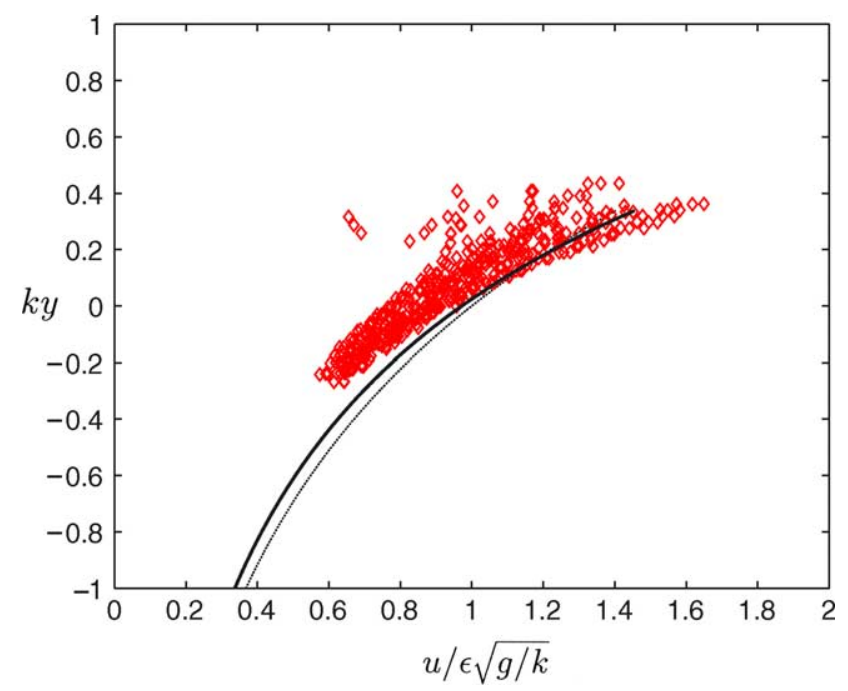

Fig. 8. Horizontal velocity profiles below wave crest. All large wave events in the random waves series $1-6$, with $\epsilon>0.3$. Solid line: Velocity profile in 'freak' wave computation with $\epsilon=0.29$. Dotted line: $\mathrm{e}^{k y}$. (Note the differences in horizontal scale.) 
The exponential profile somewhat overestimates the velocities below mean water line.

\subsubsection{Effects of a finite water depth}

A finite depth effect makes an important change of the velocity profile below the wave crest, compared to deep water: near the crest of the wave the velocity becomes increased, and below the mean water line the velocity becomes reduced. Such tendencies are observed in our random wave recordings with $2.3<k h<2.6$ and $\epsilon>0.2$.

\subsection{Comments on experimental error sources}

The strong collapse of the measurements presented in Fig. 3 indicates the high accuracy of the experimental procedure. The relatively narrowbanded wave fields in the experiments with the leading wave of a wave group and the focusing waves fit to a slowly varying modeling of the wave field. An experimental error may be quantified by the deviation from an average line through all experiments in Fig. 3a-c.

The experiments are very repeatable, with the velocity recordings showing a scatter being typically plus/minus one grid point (corresponding to $0.7 \mathrm{~cm}$; there are 40 grid points in each direction). Further, the PIV technique has a theoretical relative error that is about $2 \%$. The wave elevation is measured using wire gauges, with a relative error less than $5 \%$.

While a high accuracy of the PIV recordings generally can be obtained close to a boundary, some scatter in the velocity recordings is observed near the free surface, in some of the runs. High quality velocimetry near the free surface using small field of views will increase accuracy and reduce eventual scatter in the data. We note, however, that the maximal possible velocities induced by the waves are captured by the present experimental campaign.

\section{Discussion}

\subsection{Comparison with other work}

Baldock et al. [7] measured the velocities in steep focusing waves in deep water using LDA. The data from the time record of the steepest wave (their figure 11, case D (c)) are: $T=0.904 \mathrm{~s}$ and $\eta_{\mathrm{m}}=7.49 \mathrm{~cm}$, giving $\epsilon=0.29$, $k \eta_{\mathrm{m}}=0.34, k h=3.2$. The value of $k h$ indicates that the waves can be considered as deep water waves. Their velocities [7, figure 13c] put in nondimensional form fit excellent with the present theoretical and experimental results for deep water, see Fig. 3c. In the figure is also included the second-order model predictions from Ref. [7]. The second-order model shows a smaller velocity than the measurements. In the case studied by Baldock et al. the Wheeler stretching method [8] is quite close to the secondorder model (with $\epsilon=0.29, k \eta_{\mathrm{m}}=0.34$, results not shown). The Wheeler method significantly underpredicts the kinematics of the larger wave, with $\epsilon=0.46, k \eta_{m}=$ 0.62, as indicated in Fig. 3c.

The wave data for the largest (focusing) wave in Kim et al. [5] are: $T=1.36 \mathrm{~s}$ and $\eta_{\mathrm{m}}=15 \mathrm{~cm}$, giving $\epsilon=0.28$, $k \eta_{\mathrm{m}}=0.34, k h=2.04$. The value of $k h$ indicates that the effect of a finite water depth is important in their measurements. The velocity data for their largest wave fit well with our random wave measurements for $2.3<k h<$ 2.6 and $0.7<u / \epsilon \sqrt{g / k}<1.6$ (results not shown). The large velocity at the crest observed by Kim et al., with nondimensional value $u / \epsilon \sqrt{g / k} \simeq 2.3$, is not observed in the present measurements.

The data of the plunging wave measured by Skyner [9] are: $T=1.03 \mathrm{~s}$ and $\eta_{\mathrm{m}}=10.2 \mathrm{~cm}$, giving $\epsilon=0.3, k \eta_{\mathrm{m}}=$ $0.36, k h=2.6$. The measured horizontal velocity below crest is $2 \mathrm{~m} \mathrm{~s}^{-1}$ at maximum, i.e. $u / \epsilon \sqrt{g / k} \simeq 4$. Such a high velocity is not observed in the present deep water wave measurements.

In the large scale FULWACK experiment [2], the main purpose was to obtain velocity measurements at locations relatively high above the mean sea level using current meters. These were located at $26,16,6 \mathrm{ft}(8.2,5,1.9 \mathrm{~m})$ above mean sea level. The largest observed speed at the top current meter was $20.62 \mathrm{ft} / \mathrm{s}(6.5 \mathrm{~m} / \mathrm{s})$. The platform was in $270 \mathrm{ft}(85 \mathrm{~m})$ of water. For illustration, we assume for the moment that a wavenumber of $k=0.03 \mathrm{~m}^{-1}$ is representative for the large scale measurements (the time record of the wave elevation was not given in the paper). The corresponding wave length then becomes $2 \pi / k=209 \mathrm{~m}$, and $k h=2.6$. For the position of the upper current meter this gives $k y=0.03 \times 8.2=0.246$. Employing an exponential velocity profile, i.e. $u / \epsilon \sqrt{g / k}=$ $\mathrm{e}^{k y}$, we obtain $\epsilon \simeq 0.28$. The resulting maximal elevation of the wave becomes $11 \mathrm{~m}$ and the estimated maximal fluid velocity at the crest becomes $7.04 \mathrm{~m} / \mathrm{s}$. Similar estimates can be given with smaller or larger $k$.

\subsection{Fluid velocity relative to the wave velocity}

Kim et al. [5] measured a maximal fluid velocity being $64 \%$ of the estimated wave speed. This result may be compared with $u / c$ in the present measurements, where the nonlinear wave celerity may be estimated by $c=\omega / k \simeq$ $\sqrt{1+\epsilon^{2}} \sqrt{g / k}$. This means that $u / c \simeq \epsilon \alpha / \sqrt{1+\epsilon^{2}}$, where $\alpha$ is the nondimensional fluid velocity plotted in the figures. We obtain the following table of the maximal values of $u / c$ :

\begin{tabular}{lccll}
\hline$\epsilon$ & $k \eta_{\mathrm{m}}$ & $k h$ & $u / c$ & \\
\hline 0.29 & 0.34 & $\infty$ & 0.4 & Model, Sections 2.3 and 2.4 \\
0.29 & 0.34 & 3.2 & 0.4 & Baldock et al. [7] \\
0.46 & 0.62 & 5.0 & 0.75 & Present experiments \\
0.28 & 0.33 & 2.0 & 0.64 & Kim et al. [5] \\
0.30 & 0.36 & 2.6 & 1.14 & Skyner [9] \\
\hline
\end{tabular}


For deep water waves, the present model predictions give a value of $u$ up to $40 \%$ of the wave speed. These computations correspond directly to the measurements obtained in Ref. [7]. The present deep water wave experiments document a fluid velocity up to $75 \%$ of the wave speed $c$ (and $k \eta_{\mathrm{m}}=0.62$ ). The increase in the maximal value of $u / c$, from 0.4 in the model predictions, to 0.75 in the experiments, is in fact formidable. The latter is almost the double of the former. The plunging breaker measured by Skyner [9] has a fluid velocity that may even exceed the wave speed $\left(u / c \simeq 1.14, k \eta_{\mathrm{m}} \simeq 0.36, k h=2.6\right)$.

\subsection{Exponential profile}

In all figures we have included the nondimensional profile, $\mathrm{e}^{k y}$ where the wavenumber is determined according to the procedure in Section 2.4. The exponential profile is observed to be rather close to all the experimental observations in the top of the wave when they are put on nondimensional form. This is true provided that $k h$ is large and the fluid velocity due to a return flow beneath the waves is small. The exponential profile gives relevant estimates for all observations in deep water and $u / c$ up to 0.75 , and $k \eta_{\mathrm{m}}$ up to 0.62 . This is significantly beyond the level where the fully nonlinear model gives useful predictions.

\section{Conclusion}

The kinematics of steep water wave events has been studied comparing PIV measurements in a wave tank and theoretical/numerical predictions using a fully nonlinear wave model. A total of 62 different steep wave events have been measured in the laboratory. Several generation mechanisms have been pursued. We have studied the leading unsteady part of a wave train, waves resulting from a focusing technique and events taking place in random wave series. In the random wave experiments each of a total of six series had three repetitions showing almost no scatter of the results. The Stokes drift and a corresponding return flow beneath the wave groups is inherent in all the experiments and in the fully nonlinear computations (where the latter is effective up to breaking).

We have aimed at a general way to characterize the wave events. From the wave record (at a fixed point) we identify the trough-to-trough period, $T_{\mathrm{TT}}$ and the maximal elevation, $\eta_{\mathrm{m}}$ of an individual steep wave event. The local wavenumber, $k$ and an estimate of the local wave slope, $\epsilon$ are obtained from the equations $\omega^{2} /(g k)=1+\epsilon^{2}, k \eta_{\mathrm{m}}=\epsilon+\frac{1}{2} \epsilon^{2}+$ $\frac{1}{2} \epsilon^{3}$, where $\omega=2 \pi / T_{\mathrm{TT}}$ and $g$ denotes the acceleration of gravity. The velocity fields are put on nondimensional form dividing by $\epsilon \sqrt{g / k}$. The procedure is documented in full in Section 2.4.

Almost all of the experimental waves can be characterized as deep water waves. A strong collapse of the nondimensional velocity profiles is observed for the 13 different large leading waves events of a wave train and the 19 focusing wave events. The LDA velocity measurements by Baldock et al. [7] put on nondimensional form fit excellent with our velocity profiles, both those obtained by PIV and those obtained by the fully nonlinear theory (Section 2.3).

The kinematics in the random wave experiments with a small return fluid velocity beneath the waves conforms with the kinematics in focusing waves and the leading wave of a wave group. In some of the experiments there is a weak effect of the finite depth of the fluid, with $k h$ typically in the range 2.3-2.6. A larger velocity close to the wave crest and a smaller velocity below the mean water line than in deep water is then seen.

The experiments with the random wave fields are run so long that an additional return flow in the wave tank is induced. A return flow below the wave group generally reduces the velocity below mean water level and is responsible for an additional tilt of the velocity profile, depending on the magnitude of the return fluid velocity. This may be determined by the integrated return flow divided by the water depth below the wave, see Section 4.3.1. While the PIV technique records the instantaneous velocity field, we are unable to identify the return fluid velocity in the present experiments.

Some scatter is observed in some of the random wave experiments with a moderate wave slope. This may be due to waves of different wavenumbers that are locally interacting. The milder wave events seem to be less 'clean' than the larger waves where there is relatively little scatter in the results. Predictions using second-order model or extensions seem relevant if the sea state is moderate [15-17].

The fully nonlinear theoretical model is useful for the rather steep waves, inducing a fluid velocity $u$ of up to $40 \%$ of the wave speed. This is precisely the same level as in the experiments by Baldock et al. [7]. Both in the simulations and in the experiments by Baldock et al. the maximal wave slope was $\epsilon=0.29$. In the present experiments we have been able to push the upper value of $u / c$ (in deep water) up to 0.75 , i.e. a fluid velocity up to $75 \%$ of the estimated phase speed of the wave. The estimated value of $k \eta_{\mathrm{m}}$ is 0.62 . The focusing wave measurements [5] and [9] exhibit maximal $u / c=0.64$ and 1.14 , respectively. The corresponding estimates of $k \eta_{\mathrm{m}}$ are 0.33 and 0.36 , respectively.

A surprising result is that the exponential profile $\mathrm{e}^{k y}$ compares rather well with all measurements of the waves (put on nondimensional form) which are in deep water and when the return velocity beneath the wave group is small. The good comparison suggests that the exponential profile is quite useful for obtaining estimates of the kinematics of steep waves. It compares well with all measurements presented here, where the main focus is the velocity profile above mean water level, even for waves where the fluid velocity is up to $75 \%$ of the estimated wave speed. We note that the exponential profile should be used in connection 
with the procedure determining the local wave slope and wavenumber outlined here.

The Wheeler stretching method, widely used in engineering practice, replaces the vertical coordinate $y$ by $y^{\prime}=h(y-\eta) /(h+\eta)$. For large water depth this means $y^{\prime}=y-\eta$. It is easily seen that our results do not support the Wheeler stretching method. In fact, for the steepest waves investigated here, the Wheeler method predicts a particle velocity under crest being about one-half of the true velocity, see Fig. 3c. On this point our results are closer to a velocity profile that is linearly extrapolated above the mean water line. Longridge et al. [6] compared velocities from LDA measurements of random wave series with theoretical models including linear extrapolation, Wheeler stretching and a hybrid wave model. They found that the former was the superior one for the range of wave slope under investigation $(\epsilon=0.11-0.19)$. For this range, the expansion $\mathrm{e}^{k y}=1+k y$ is valid, indicating mathematically the usefulness of a linear extrapolation of the velocity field above mean water line. This conclusion has been supported by second-order computations [24] comparing with irregular wave experiments [4]. A linear extrapolation underestimates the kinematics of steep wave events, however.

The present experiments focusing on the velocity below crest, confirm the value of irrotational flow computations of the entire velocity field below steep waves (which are now rather quick).

\section{Acknowledgements}

This work was conducted under the Strategic University Programme 'General Analysis of Realistic Ocean Waves' funded by the Research Council of Norway. The technical assistance in the Hydrodynamics laboratory by $\mathrm{Mr}$ Arve Kvalheim and $\mathrm{Mr}$ Svein Vesterby are gratefully acknowledged.

\section{References}

[1] Buckley WH, Stavovy AB. Progress in the development of structural load criteria for extreme waves. Proceedings of the Extreme Loads Response Symposium. The Ship Structure Committee and the Society of Nav. Arch. and Mar. Engng. (SNAME), USA; 1981.

[2] Forristall GZ. Kinematics in the crests of storm waves. Proceedings of the 20th Coastal Engineering Conference, Taipei, Taiwan; 1986. p. 208-22.

[3] Skjelbreia JE, Gudmestad OT, Ohmart RD, Berek G, Bolen ZK, Heideman JC, Spidsoe N, Tørum A. Wave kinematics in irregular waves. OMAE I-A, Offshore Technology; 1991.

[4] Skjelbreia JE, Tørum A, Berek E, Gudmestad OT, Heideman JC, Spidsoe N. Laboratory measurements of regular and irregular wave kinematics. NorgesTekniske Universitetsbibliotek. Eske 604; 1991.
[5] Kim CH, Randall E, Boo SY, Krafft MJ. Kinematics of 2-D transient water waves using Laser Doppler Anemometry. J Waterway, Port, Coastal Ocean Engng 1992;118(2):147-65.

[6] Longridge JK, Randall RE, Zhang J. Comparison of experimental irregular water wave elevation and kinematic data with new hybrid wave model predictions. Ocean Engng 1996;23:277-307.

[7] Baldock TE, Swan C, Taylor PH. A laboratory study of nonlinear surface water waves. Philos Trans R Soc Lond, A 1996;354:649-76.

[8] Wheeler JD. Method for calculating forces produced by irregular waves. Proc First Annual Offshore Technol Conf (Houston) 1970;1: $71-82$.

[9] Skyner D. A comparison of numerical predictions and experimental measurements of the internal kinematics of a deep-water plunging wave. J Fluid Mech 1996;315:51-64.

[10] Dold JW, Peregrine DH. An efficient boundary-integral method for steep unsteady water waves. In: Morton KW, Baines MJ, editors. Numerical Methods for Fluid Dynamics II. Oxford: Clarendon Press; 1986. p. 671-9.

[11] Peregrine DH. Computations of breaking waves. In: Tørum A, Gudmestad OT, editors. Water wave kinematics. Dordrecht: Kluwer; 1990. p. 475-90.

[12] Pierson WL, Banner ML. Aqueous surface layer flows induced by microscale breaking wind waves. J Fluid Mech 2003;479:1-38.

[13] Gudmestad OT. Measured and predicted deep water wave kinematics in regular and irregular seas. Marine Struct 1993;6:1-73.

[14] Longuet-Higgins MS, Stewart RW. Changes in the form of short gravity waves and tidal currents. J Fluid Mech 1963;8:565-83.

[15] Zhang J, Chen L, Ye M, Randall R. Hybrid wave model for unidirectional irregular waves. Part 1 . Theory and numerical scheme. Appl Ocean Res 1996;18:77-92.

[16] Zhang J, Yang J, Wen J, Hong K. Deterministic wave model for shortcrested ocean waves. Part I. Theory and numerical scheme. Appl Ocean Res 1999;21:167-88.

[17] Spell CA, Zhang J, Randall R. Hybrid wave model for unidirectional irregular waves. Part 1. Comparison with laboratory measurements. Appl Ocean Res 1996;18:93-110.

[18] Fenton JD. The numerical solution of steady water wave problems. Computers Geosci 1988;14(3):357-68.

[19] Jensen A, Sveen JK, Grue J, Richon J-B, Gray C. Accelerations in water waves by extended particle image velocimetry. Exp Fluids 2001;30:500-10.

[20] Clamond D, Grue J. A fast method for fully nonlinear water-wave computations. J Fluid Mech 2001;447:337-55.

[21] Clamond D, Grue J. On efficient numerical simulations of freak waves. Proceedings of the 11th International Offshore and Polar Engineering Conference and Exhibition, Stavanger; June 17-22, ISOPE 2001.

[22] Clamond D, Grue J. Interaction between envelope solitons as a model for freak wave formations. CR Acad Sci Paris 2002;330:575-80.

[23] Taylor PH. On the kinematics of large ocean waves. Proc Behav Offshore Struct Conf (Lond) 1992;1:134-45.

[24] Stansberg CT, Gudmestad OT. Non-linear random wave kinematics models verified against measurements in steep waves. Proc Offshore Mech Arctic Engng Conf (OMAE. Florence) 1996;15-24.

[25] Moe G, Arntsen $\varnothing$, Gjøsund SH. Wave kinematics based on a Lagrangian formulation. In: Zhang J, editor. Proceedings of the Ocean Wave Kinematics, Dynamics and Loads on Structures. ASCE Symposium, Houston,TX; 1998. p. 56-63.

[26] Grue J, Bjørshol G, Strand $\varnothing$. Higher harmonic wave exciting forces on a vertical cylinder. Preprint. Norway: Department of Mathematics, University of Oslo; 1993.

[27] Grue J, Huseby M. Higher harmonic wave forces and ringing of vertical cylinders. Appl Ocean Res 2002;24:203-14. 